Journal of Teacher Education for Sustainability, vol. 19, no. 1, pp. 147-163, 2017

\title{
Bullying and Victimisation Dynamics in High School: An Exploratory Case Study
}

\author{
Lucy M. George Lekunze \\ Newark Public Schools, United States of America \\ B. Ivan Strom \\ University of Phoenix, United States of America
}

\begin{abstract}
Bullying is a worldwide concern and erroneous perceptions of the phenomenon could underscore unsustainable interventions. The purpose of this qualitative exploratory case study was to examine, in-depth, how some high school teachers from two schools in New Jersey perceived student bullying. The primary research question was: What perceptions do teachers have about student bullying? The main data were from face-to-face interviews with 14 teachers who answered semi-structured, open-ended questions. Secondary data originated from physical artefacts. The data analysis consisted of four phases. Inductive analysis allowed for the composition of individual cases. Cross-case analysis allowed data classification into three main areas of inquiry aligned with the three secondary research questions (a) bullying and victimisation dynamics as perceived by teachers, (b) adequacy of current interventions, and (c) adequacy of teachers' professional development for bullying mitigation. The data unveiled inconsistencies between causes of bullying and interventions. It culminated in recommendations for leadership and suggestions for future research.
\end{abstract}

Keywords: bullying, victimisation, dynamics, consequences, intervention, consistency, adequacy, professional development, sustainability.

\section{Introduction}

Bullying, as defined by Bennett (2014), Jones and Augustine (2015), Malecki, Demaray, Coyle, Geosling, Rueger, and Becker (2015) and Wilder (2014) is a repeated maltreatment of a victim by one or more perceivably stronger others; bullying threatens the peace, tranquility, health, and general welfare of members of societies. Jones and Augustine reiterated the need to eradicate bullying because continual victimisation can cause longterm physical illness, mental health problems, interpersonal, emotional, and self-esteem issues for targets, the surrounding peer group, and perpetrators. Chronic untreated symptoms of bullying and victimisation can undercut the scholastic competence, development, and general welfare of a child (Margeviciute, 2016). Scholastic difficulties affect self-esteem, ignite antisocial behaviour, promote peer rejection, and lead to self-with- 
drawal (Leeuwis, Koot, Creemers, \& van Lier, 2015). Victimisation can carry several connotations. For instance, people can experience victimisation from random street mobbing, robbery, bad relationships, and corrupt governments. In the context of the present research, victimisation refers to oppression from bullying only.

The problem is that erroneous perceptions of bullying and victimisation could undermine intervention initiatives, as most currently used anti-bullying strategies are reactive disciplinary sanction on the bully (Rigby, 2014). Rigby believed that more restorative practices like peer mediation, which equips students to take proactive steps to diffuse a bullying encounter before it escalates, is more realistic. Rigby described the ability of students to engage in a naturally creative manner to dissolve a bullying altercation without the need for adult involvement (oftentimes, with harsher solutions) as sustainable; not involving any adverse outcomes to the perpetrator or the victim who are both minors mandating a restorative environment. Considering that bullying is an inherent part of child development used to impose social hierarchies (Lantos \& Halpern, 2015), sensible or sustainable solutions would be those directed at the antecedents instead of the symptoms of bullying. Inadequately addressed symptoms of bullying/victimisation, including depression and culminate in emotional instability (Jones \& Augustine, 2015). Emotional instability is a condition incompatible with learning that decreases school satisfaction, creates anger, invokes truancy, lawlessness and scholastic regression in youths (Golmaryami et al., 2016).

Data from this qualitative exploratory case study unveiled how some high school teachers in New Jersey felt about bullying and victimisation as it related to the adequacy of current intervention etiquettes, contributing additional insight to existing knowledge. As mediators who interact daily with students, teachers contributed valuable information for managing bullying and victimisation issues. Students with firsthand experience as bullies or victims initially attracted attention as possible information-rich participants in this study; however; the ethical concerns and bureaucratic loopholes associated with obtaining authorisation to interview minors about emotionally sensitive issues prompted a change in decision.

The next information-rich source closest to students were teachers spending significant amounts of time observing behaviours and interacting with students daily. That explains the choice of teachers as research participants in this study. Most explored prior studies about school bullying took place in public elementary and middle schools and some authors recommended a replication in public high schools. Such recommendations inspired the choice in this study to interview public high school teachers addressing sampling limitations and gaps in teacher input (Charmaraman, Jones, Stein, \& Espelage, 2013). This study addressed the data limitations from teachers by employing a qualitative exploratory case study design, which entailed data gathering from high school teachers as experienced from the second-person viewpoints and triangulated data collection procedures that guaranteed adequate population representation (Leeuwis et al., 2015; Charmaraman et al., 2013).

The primary research question was: What perceptions do teachers have about student bullying? In addition to the primary research question, the following secondary research questions enhanced the process of understanding how some high school teachers in Essex County, New Jersey perceive student bullying: (1) What are the bullying dynamics as perceived by teachers? (2) How has professional development (if any) helped teachers in bullying mitigation? (3) What forms of interventions do the schools use and what are 
teachers' perceptions of the adequacy of current interventions? The nature of the teaching job entailing day-to-day interaction of teachers with students predisposes teachers to direct witnessing of bullying and victimisation incidents and student reporting. Teachers therefore represented an information-rich source from which the investigator could extract substantive insight into the bullying phenomenon. The insight unveiled suggestions for interventions with more practical potential, enhanced professional development for school personnel, and the refinement of school policies.

Leaders in education should care about solving the problem because of the veracity and criticality of bullying in undermining the safety and welfare of youths (Austin, Reynolds, \& Barnes, 2016). Diers (2013), Douglas (2014), and Saldana (2015) expressed concerns about the limited attention child safety issues received from the scientific community despite a potentially adverse repercussion on child development. An increased initiative to reach the most marginalised children is needed to close an equity gap (Diers, 2013). Hertz et al. (2015) projected that childhood exposure to bullying was an adverse experience associated with adult asthma, smoking, sexually transmitted disease, obesity, substance use, depression, and sleep disturbances. Additional literature supports that bullying is a health-complicating issue needing further research to guide reforms in school administrative decisions and societal policy (Hertz et al., 2015; Herge, La Greca, \& Chan, 2016). This study contributed to addressing such gaps in knowledge necessary for leaders to design more feasible intervention programmes for bullying and victimisation. The purpose of this qualitative exploratory case study was to examine how some high school teachers in New Jersey perceived bullying. This study contributed further insight into perspectives of teachers about personal experiences with bullying and sufficiency of existing peer support structures to improve school, community, and home environment.

Literature review unveiled a wide coverage of the problem of bullying and victimisation. On a national scale, approximately 160,000 students were absent from school daily in the United States to avoid victimisation by bullies (Nash, 2012; Kearney \& Graczyk, 2014; Kramer, 2015). Chui and Chan (2015) speculated that bullies had low self-control with higher risks of involvement in criminal activities. Victims of bullying suffer negative consequences, such as depression, anxiety, academic demotivation, and the possibility of dropping out of school (Lianget al., 2014). According to Nash, aggravated depression in some victims results in suicide. Wilder (2014) and Bennett (2014) noted that victims of bullying oftentimes resorted to viciousness in vengeance against oppressors. Such viciousness manifested itself in recent school assassinations in the United States ("Bullying and Statistics", 2012). In addition to the unprecedented degrees of unaddressed psychological health issues in schools, there is also less likelihood of children suffering from different forms of disabilities learning productively because of bullying (Bear et al., 2015).

Literature sources project significant connections among positive role modelling, levels of empathy, and social support exhibited toward youths by their entourage and resultant emotional responsiveness (Wilder, 2014). For example, Hertz et al. (2015) demonstrated that childhood exposure to adverse experiences contributed to youth misbehaviour. Teicher and Parigger (2015) stipulated a connection between home condition and aggressive behaviour in teenagers. Parents contribute enormously in shaping child behaviour. According to the Bandura's theory on shared learning and attachment, the environment, including school, family, and peers significantly influence child behaviour (Judkins, 2014; Deaton, 2015). Children tend to admire and trust adults who live by 
their words. Garthe, Sullivan, and Kliewer (2015), also Ciudin and Turliuc (2016) speculated that because children had the tendency of internalising everything (both negative and positive), parental conflict could compromise child self-concept.

Based on Bandura's theory on shared learning and attachment, children spontaneously copy behaviours from primary entourage or immediate circle, which include parents, siblings, extended family, peers, teachers, school officials, and more (Deaton, 2015; Swearer, Wang, Berry, \& Myers, 2014). Deaton believed that positive parental attachment, during which parents establish healthy relationships with their infants, could influence children's future interaction and reaction toward others. Implicitly, the quality of parental care and guidance could influence children's tendency towards delinquency. That makes one wonder to what extent societal structures and systems can shift behaviours that have been developed early in a child's life.

Many reports have identified connections between parental neglect and youth delinquency (Saldana, 2015; Paat, 2013). Some reports attributed the inadequacy of most community programmes, including Corrections Education (CE) initiatives designed to reform troubled youths to their standardisation in harmony with state conventions (Jones \& Augustine, 2015; Kueny \& Zirkel, 2012). Standardised interventions, which typically are based outside family settings with limited parental role-playing, often concentrate on developing delinquent youths mentally instead of eliminating original triggers of their delinquency, which include negative adult examples, poor moral orientation, dysfunctional families, adverse school climate, and community environments. Because parents, teachers, and community play significant roles in determining the personalities of their children, attempts to resolve school bullying without adequate teacher involvement could reflect lack of common sense.

Recognising connection between adverse environment and juvenile misbehaviour (Deaton, 2015; Paat, 2013; Swearer, Wang, Berry, \& Myers, 2014) and due to inconsistencies between CE initiatives and contributors to bullying (Jones \& Augustine, 2015), there appears to be unknown or poorly understood elements vital to address the problem of bullying. This qualitative exploratory case study responded to the additional data need by enriching societal comprehension of bullying-victimisation from the lens of the teacher. New information from the study could prepare teachers, school administrators, and school policy makers to address better bullying-victimisation issues at every school level.

The authors used Bronfenbrenner's ecological system theory as the conceptual framework for the study perceiving the mainstream school as an ecological system, in which components interact constantly influencing individual identity. The authors draw on the insights of Bronfenbrenner's ecological system theory that bullying behaviour results not only from individual characteristics of a juvenile bully but also from indirect influence of his or her entourage and general environment. Some indirect factors influencing bullying interactions include cultural factors, parents, extended family, teachers, school officials, peers, community factors, physical characteristics of school environment, school anti-bullying policies, and school atmosphere, for instance, student perceptions of selfsafety (Bronfenbrenner, 1974/1977/1986/1979/1988/1993; Paat, 2013). Support for the practicality of Bronfenbrenner's ecological systems theory in moderating juvenile personality abound (e.g., Hong \& Garbarino, 2012; Huang, Foster, \& Brooks-Gunn, 2013; Hong \& Espelage, 2013; Patton, Hong, Williams, \& Allen-Meares, 2013; and Espelage, 2014). As the ecological systems play significant modelling roles in driving 
bullying-victimisation interactions, anti-bullying interventions not assuming equally holistic integrated approaches by partnering with social ecological stakeholders playing critical functions in shaping how children will act over their teenage life courses, appear unrealistic.

\section{Method and Design}

The research population consisted of the perceptions of high school teachers in Essex County, New Jersey. The data were from a sample of 14 high school teachers in two cosmopolitan public high schools in Essex County, New Jersey. The scope was limited to two schools whose identities remain concealed for privacy and confidentiality purpose. The initial plan included five public high schools. All 32 public high schools in Essex County, New Jersey received mailed envelopes containing a letter of introduction and permission forms for school superintendents or principals to sign indicating an endorsement of the project. Only the principals and superintendents from the two high schools that participated in this study signed and returned the permission forms leaving us no other choice than to work with the two cooperating schools. The choice of teachers as self-reporting research participants stemmed from three motivating factors. (1) The literature gap related to teachers' input in deciphering the bullying dilemma (Charmaraman, Jones, Stein, \& Espelage, 2013). (2) Teachers are predisposed to first-hand and secondhand witnessing of bullying as they interact directly with students on almost daily basis. (3) Ethical concerns that juveniles will re-experience their undesirable pasts by discussing self-experiences as bullies or victims led to the choice of teachers instead of students as research participants although students could be information-richer than teachers.

The method for this study was qualitative and the design was an exploratory case study with face-to-face interviews conducted in teachers' lunchrooms using open-ended semi-structured questionnaires (Appendix 1) implemented following rigorous guidelines with feedback sessions. The method appeared appropriate in the context of broader scholarly literature. Qualitative method and case study design have wide application in researching bullying behaviour (e.g., Johnston et al., 2014; Pister, 2014; Singh, 2013; Ramirez, 2013) though not without inherent limitations often from small data size (Rostkowski \& Singh, 2015). The open-ended questions permitted interviewees to apply their subjective visions, judgments, logic, and reasoning to determine their own options of unrestricted responses with advantage of generating an in-depth insight into personal life experiences. The open procedure espoused autonomy, a highly valued inter-human quality unattainable with quantitative methods. The semi-structured open-ended questions (with direct evidential connection to the original research questions) engendered a broad discussion approach that enhanced the researchers learning from participants through exploration to fill in literature gaps about bullying; a poorly understood phenomenon (Enlgander, 2012).

The authors chose a qualitative method because quantitative methods require statistical data derived from surveying pools of research participants representing numeric data from structured questionnaires with predetermined closed-ended questions limiting interviewees' answers (Koskey, 2016), which cannot permit unbiased capturing of bullying dynamics. Pister (2014) believed that an objective way to capture salient relationships in cryptic behaviours was to integrate data reflecting the opinions and insights of research 
participants as attainable through qualitative methods employing a case study design and semi-structured open-ended interviews.

The case study design was appropriate because case studies involve a diligent examination of specific individuals, entities, or units to appraise unique patterns of thinking or behaviour as subjects narrate and describe their subjective experiences from varying perspectives (Singh, 2013). Case studies or case reports are processes of data gathering in which researchers consider the development of a specific phenomenon or issue within a particular real-life context over time, using particular instances of individual units to illustrate and analyse concepts intensively in an explanatory, descriptive, or exploratory manner.

Following Palinkas et al. (2015) the authors of the present research used a purposeful sampling to explore the perceptions of handpicked experienced information-rich teachers (nominated by school officials) about their involvement with student bullying. The authors adopted a technique of data triangulation that accomplished breadth, depth, minimized bias, and fostered validity (Leeuwis et al., 2015; Charmaraman et al., 2013). Data arose from different sources employing different data gathering tactics to accomplish data saturation. Data saturation, according to Rostkowski and Singh (2015), is a technique of data triangulation through which analysts use independent bits of information obtained through face-to-face conferences with different decisively selected respondents from different sources to gain a better grasp of a poorly understood or unknown occurrence. The different sources of data in this exploratory case study were the subjective utterances of high school teachers from two public high schools (primary data source) and unrestricted physical artefacts with open public access (secondary data source). For ethical reasons, secondary data sources excluded individual teacher diary and official school records requiring access permit.

According to Dehkordi, Babashahi, and Irajpour (2016), in qualitative studies in which probabilistic sampling is impossible, investigators could justify sample size by employing different data gathering tactics to attain data saturation when further interviews cannot yield any new information. Data saturation attainable through interviews employing diverse data gathering tactics counteracts possible sampling biases that undermine validity, trustworthiness, and dependability (Mason \& Ide, 2014). Some data gathering tactics employed by the authors included confidentiality statements, signed informed consents, and alphanumeric data coding, which excluded participants' real names (e.g., F1, F2, M1, M2, etc.). The authors also used semi-structured openended questions, asked the same interview questions repetitively in different forms/ structures to enhance consistent and comprehensive responses, took clarifying questions, offered feedback sessions, and recruited six independent neutral peer data reviewers.

In the analytical procedure, the authors used Patton (2002) and Snelgrove (2014) as guides by embracing an ideographic position mandating us to reread, audit track by cross-examining notebooks, voice recordings, and artefacts, and analysing each transcript comprehensively before proceeding to the next, treating each script independently in an inductive manner. Data analysis proceeded simultaneously with data collection employing the method of Patton, entailing data reduction, unitisation, classification, displays, extrapolation, and verification. The authors represented related topics in a table of superordinate themes for each interviewee before proceeding to the next. The authors also compared the themes for each interviewee across the dataset to identify emerging concepts as new themes, repetitions of existing theme, and super-ordinate themes cutting across the entire 
sample. The authors replicated the procedure for each of the 14 interviews going back and forth comparing new super-ordinate themes from each interview with those extracted from previous interviews. According to Snelgrove (2014), this bottom-up approach allowed us to explore all 14 sets of interviews without compelling conventional theories on the data.

As recommended by Zamawe (2015), the authors used NVivo10 analytical software to subject the amorphous qualitative data into a rigorous process of analysis, including exploration, coding, indexing, management, and interpretation making sense of narrative information in a computerised database. With the transcripts titled as sources in the coding reports, NVivo10 sorted the transcripts alphabetically by the unique participants' alphanumeric identifiers. The coding process connected passages in the data to develop categories, themes, ideas, organised nodes to contain the ideas emerging from the sources as mirrored in the themes, and identified references to emerging concepts (Bhatnagar, \& Das, 2014; O’Neill, Calder, \& Allen, 2014). NVivo10 coding entailed the nomination of a portion of a source representing transcripts categorised by research participants relating to a node. The data manager imported the transcripts for participants identified as F1, F2, M1, M2, and more into an NVivo10 project.

An alignment of teachers' responses to the three secondary research questions projected the extent to which the teachers answered the original research questions. Categorisation into three sections (aligned with the three secondary research questions) and 19 facets provided clear pictures of the data. The various facets reduced further into sub-facets enhanced further data clarity. The authors presented the three sections and 19 facets in graphic formats to demonstrate what each participant said with respect to each or a combination of components. Elimination of less frequent ones enhanced further data clarity. The data could be trusted because the study was strictly exploratory with no intent to verify or prove an existing hypothesis, theory, or the researchers' personal judgments, opinions, and assumptions. The data were an undistorted representation of real life experiences of the teachers shared face-to-face and presented in the partakers' own words. Exclusion of personal biases and fostering of rigor occurred through the bracketing and inductive approaches described by Patton (2002) and Snelgrove (2014) entailing the following steps. (1) Treating each transcript in an inductive, open-ended, and exploratory manner without imposing any established theories on the data. (2) Identifying key expressions in respondents' statements that related to bullying. (3) Meticulously interpreting the meanings of the expressions related to bullying. (4) Interpreting expressions and meanings from participants' standpoint. (5) Diagnosing what the expressions unveiled about an observer of violence. (6) Determining what a practical intervention for bullying would entail in context of the repetitive meanings.

Six steps that fostered quality and validity included the following. (1) Adequate documentation of information in the course of data collection. (2) Organisation or categorisation of the data into emerging concepts. (3) Connection of the data to demonstrate relationships between emerging concepts. (4) Legitimisation and corroboration that entailed consideration of alternative explanation of concepts, disaffirmation of evidence, and consideration of adverse cases. (5) Representation of the findings in a reporting format that aligned with research questions. (6) Teachers' responses transcribed verbatim from voice recordings prevented possible alteration of interview responses.

To reinforce dependability, the authors used the Audit Track method (Palinkas et al., 2015) entailing purposive selection of information-rich interviewees knowledgeable 
about bullying, pilot pre-testing of interview questions, and analysis of research procedure to verify consistency with outcome (Morgan, Leatzow, Clark, \& Siller, 2014). The audit track method also entailed recording of interviews, transcription, verification of transcription by informants, employing a software such as NVivo10 to manage data, comparing transcriptions with optional independent reports/narratives such as published articles, posters, and websites for consistency (Geller et al., 2015). As Jiang, Chen, Ouyang, and Li (2015) noted, such tenacity in data comparison enhances data quality optimisation.

The authors used self-reports reflecting teachers' encounters with bullying (from a second-third person standpoint), which had some merits over self-reports by bullies and victims (from a first-second person standpoint) who could intentionally falsify statements to implicate rival peers or to avoid punishment. However, first-person narratives have impassable validity merits, if undistorted but the authors evaded students for priorexplained ethical reasons. Validity problems associated with self-reports include the possibility of over and under reporting (Baly, Cornell, \& Lovegrove, 2014). Participants could exaggerate their experiences to project crisis for attention purpose or they could under-represent the severity of their experiences. The authors used the audit track method discussed earlier to verify responses for consistencies. Self-reports are advantageous in permitting participants to describe their own lived experiences instead of analysts making inferences from utterances or body language. An overarching limitation of this study was the inclusion of two schools only because of unwillingness of school superintendents to allow access. As such, it is possible that some of the schools with higher student bullying or victimisation rates may be under-represented in this study.

\section{Results}

A cross-case analysis of the teachers' perceptions of bullying resulted in seven identified major themes prompting the authors to organise or categorise the data into the following seven emerging concepts. (1) Teachers differed in their understanding of the definitions of bullying. (2) Teachers expressed concern about the academic and personal consequences of bullying. (3) Teachers did not differ in their approaches to bullying. (4) Teachers felt inadequately prepared to consistently address bullying issues. (5) Teachers recommended that additional school staff focus on bullying prevention. (6) Teachers expressed the need for additional school programmes for students that focused on bullying prevention. (7) Teachers voiced the opinion that teachers should be invited to contribute to bullying prevention policy.

For definition, teachers' general idea was that bullying is a very serious problem and without exception, every aggressive action constitutes bullying. For example, in response to question eight (Appendix 1), Mr.F6 stated, "Anything that is leaving another person feeling powerless, to me is bullying." When the authors reworded and repeated the question the second time, Mr.F6 also reworded his response saying, "Any kind of aggressive behaviour must be addressed immediately." When the authors reworded question eight the third time petitioning a new response, Mr.F6 again modified his response this time saying, "I inquire about any aggressive behaviour as it is happening because you can never be sure." Note that all three responses by Mr.F6 to the three versions of question eight asked repetitively in different formats (to check for consistency, validity, trustworthiness, and dependability) insinuated that every aggressive behaviour should be construed as bullying without exception. 
The most reported forms of bullying by the teachers were physical and verbal forms. The other forms with lesser frequencies included cyber bullying, peer pressure, snatching and confiscating of items from victims, prejudice, discrimination, being overly judgmental, and constant negative critiques. Teachers attributed verbal bullying mostly to girls and physical bullying mostly to boys. However, both genders indulge in different forms of bullying behaviours. In the context of evolution, most teachers believed that bullying behaviours did not change, that bullying was always part of school tradition and would continue to be. The teachers asserted that bullying behaviours remained unchanged, but the types of bullying behaviours with electronic forms were increasing as technology advanced.

The consequences of bullying were numerous with top on the list being suicide. Most participants believed bullying is very serious because of the injury factor associated with it, which in severe cases could instigate suicide. The leading motivations for bullying were the Internet, playing improperly, and joking improperly. The teachers thought that most bullies had bullying parents as negative role models. Other motivations for bullying included a compromising culture, lack of accountability, and the tendency of some students attempting to protect their self-esteem and dignity by concealing initial victimisation thereby encouraging their bullies to reinforce their negative actions without consequences. Negligently attempting to resolve the bullying issue from the surface without digging deep into the roots is a factor of failing intervention, according to some teachers. The root of the problem appears to stem as far back as the slavery era with one teacher arguing that the country was founded on bullying. The data revealed diversities of traits displayed by bullies (e.g., secure, financial extortion and gang involvement) and victims (e.g., suicide, insecure, and fear). Examples of aggressive behaviours not perceived as bullying showed that teachers mostly considered and treated every aggressive action as bullying.

Teachers did not differ in their approaches to bullying. Their self-reports indicated that teachers employed numerous forms of interventions top of which was intercession to stop bullying incidents as they were happening. For instance, in response to question nine (Appendix 1), Mr.F5 stated, "The teachers have to stop the bullying encounter on the scene, then, notify or report the incident to the student counselors. Teachers have to fill out the Harassment Intimidation and Bullying (HIB) forms and refer the students involved to the counsellor and the counsellor takes it from there and provides the right intervention to mitigate the problem." Teachers believed that current interventions were inadequate because they were mostly standardised despite the fact that every bullying encounter and every individual involved in a bullying incident were unique requiring unique intervention approaches. School climate reportedly is negative, boosting effects of bullying and victimisation. The suggestions for improvement of current interventions pertained to both educational authorities and future research.

Teachers felt inadequately prepared to consistently address bullying issues. The most celebrated form of teacher training was the HIB law. Some teachers said although they passively applied their professional developmental skills in bullying mitigation, they mostly relied on their common sense of judgment and on-the-job experiences in bullying mitigation because professional development skills were defective and incapable of serving as panacea in every situation. The main challenges teachers face is the bullying or victimisation occurring outside their range for which they have limited control. 
Teachers recommended that additional school staff should focus on bullying prevention. If a consistent routine of anti-bullying culture is reinforced in classrooms, hallways, cafeterias, and playgrounds, it could deter bullies. If not only teachers and administrators but also every adult parading the school premises should act as watch dogs, bullies could dissuade further.

Teachers expressed the need for additional school programmes for students that focused on bullying prevention. Data showed that students did not feel free reporting victimisation. This tendency could reverse if additional school-wide programmes such as the advisory ones providing opportunities for one-on-one dialogue between students and a trusted adult became available.

Teachers voiced the opinion that teachers should be invited to contribute to a bullying prevention policy. Data indicated that teachers were not involved in policy development. Among the long list of suggestions for improvement of teachers' roles in bullying mitigation were awareness raising, culture change, and parental involvement.

\section{Discussion}

The primary research question pertained to the perceptions of teachers about student bullying. Corresponding answers to the three secondary research questions enhanced understanding of the high school teachers' perception of bullying. The extensive data analysis unveiled answers to the questions presented in the form of afore-listed seven major themes.

The teachers' perceptions of bullying as a very serious problem were consistent with background knowledge (Bullying and Statistics, 2012; Hoglund, Hosan, \& Leadbeater, 2012; Huang, Hong, \& Espelage, 2013; Jansen et al., 2012; Kramer, 2015; Margeviciute, 2016; Leeuwis et al., 2015). Their viewpoint that every aggressive action (irrespective of its repetitive status) is bullying without exception was inconsistent with prior views (Bennett, 2014; Jones \& Augustine, 2015; Malecki et al., 2015; Van Fleet \& Van Fleet, 2012; Wilder, 2014). Observations that students exhibited different types of bullying behaviours with the most commonly reported being physical and verbal aggression were consistent with published reports (e.g., Schneider, Odonnell, \& Stueve, 2012). Although teachers reported physical and verbal aggression in both genders, boys displayed more physical and girls more verbal aggression, a gender disparity with background support (e.g., Topcu \& Erdur-Baker, 2012).

Teachers projected that electronic bullying continued to increase under the enhancement of advancing technology and societal concession. Although most participants believed that bullying behaviours did not evolve (consistent with Nash, 2012), they observed a change in practice from physical to electronic forms of bullying with advancing technology, which was supported by prior literature sources (De Souza \& McLean, 2012; Wilder, 2014). The data showed that bullying and victimisation, which reportedly occurred daily in the schools, represented serious concerns to students, teachers, and school administrators. Consequences varied with most severe being suicide consistent with prior reports (e.g., Kramer, 2015). Motivation for bullying also varied and consistent with empirical report (e.g., Ringwalt \& Shamblen, 2012; State of New Jersey, 2013), adverse school climate was foremost.

Some of the characteristics of bullies and victims mentioned by the participants such as physically stronger and physically weaker respectively were findings common 
in prior literature e.g., observations by Huang et al. (2013) that individual characteristics and personality traits influence bullying and victimisation behaviours. Intervention protocols mentioned by the teachers included the HIB law, social worker mediation, the debate club, and the advisory. The heightened sense of responsibility and willingness by teachers to intervene in bullying and victimisation situations (despite some lack of confidence) and the perceived inadequacy of current interventions were documented in past literature (e.g., Caldwell, 2012). Yoo (2016) projected that adequate professional development enhanced teacher efficacy. The teachers believed the HIB (a celebrated intervention and teachers' professional buttresses) was inadequate partly because of its (a) annual nature, (b) standardisation, (c) cumbersome reporting process, (d) slow response, and (f) reactive instead of proactive nature. Additional ideas such as school social programmes (e.g., debate and advisory) appeared practical but not always fully functioning.

The school climate by virtue of its open-door policies can potentially enhance students' levels of comfort with reporting victimisation. The teachers' observations that negative school climate can exacerbate bullying led to recommendations to enhance anti-bullying school climate and involvement of teachers more in policies. The report showed that although teachers played a frontline role in bullying mitigation, their voices might remain unheard from the school level to the state level. Policy makers would hardly grasp the full dynamics of these complex issues without an opportunity to appreciate such real-life experiences. The wise direction to enhance leadership choices is apparently a partnership that integrates students, teachers, school personnel, parents, and community voices (Deaton, 2015; Bronfenbrenner, 1993; Jansen et al., 2012; Swearer et al., 2014; Williams \& Kennedy, 2012; Zwierzynska, Wolke, \& Lereya, 2013).

\section{Conclusion and Recommendations}

Bullying dynamics as perceived by teachers has raised concern about current reactive anti-bullying punitive initiatives, which do not equip children to creatively diffuse unwelcomed peer advances offsetting possible adverse outcome to both the bully and victim; a sustainable solution that excludes adult intervention needs. The inconsistencies between causes of bullying and interventions unveiled by the data resulted from misconceptions of bullying and victimisation. If prior authorities couldn't extenuate bullying, the likelihood of contemporary authorities succeeding is questionable considering that bullying is an inherent component of child development. The implication is that school leaders could re-direct efforts from attempts to eradicate to more sustainable attempts to foster positive coping skills in victims.

Educational authorities should note that a modern youth society is very dynamic exposing children to different types of orientations, behaviours, thinking styles, and differential mastery levels necessitating an adaptive individual-specific strategy in behavioural management contrary to the standardised protocols reported in this study. The conceptual framework supporting this research mandates a holistic intervention approach that should include students, parents, school staff, policy makers, communities, and other stakeholders involved in the life of a child to establish consistency within and without school environments respecting expectations for appropriate behaviours and consequences for inappropriate behaviours.

Professional development needs reinforcement to adequately prepare teachers for bullying mitigation. It is mandatory for educational authorities to adopt an integrated 
approach incorporating teachers' input in planning HIB anti-bullying law, annual workshops, and other conferences on conflict management, appeasement, peer mediation, and bullying management. HIB training should help teachers not only to identify bullying scenarios effectively but also to conduct proper management of bullying and victimisation through proactive removal of the motivational forces of juvenile aggression.

One limitation of this study was the inclusion of two high schools only in New Jersey with possibilities that some schools with higher student bullying rates might be under-represented. Future studies could address this limitation by examining more than two schools. Considerations of schools outside New Jersey could be of additional value.

\section{References}

Albdour, M., \& Krouse, H. J. (2014). Bullying and victimization among African American adolescents: A literature review. Journal of Child \& Adolescent Psychiatric Nursing, 27(2), 68-82. doi: 10.1111/jcap.12066

Austin, S. M., Reynolds, G. P., \& Barnes, S. L. (2016). School leadership and counsellors working together to address bullying. Reading Improvement, 53(4), 188-194.

Baly, M. W., Cornell, D. G., \& Lovegrove, P. (2014). A longitudinal investigation of self- and peer reports of bullying victimization across middle school. Psychology in the Schools, 51(3), 217-240. doi: 10.1002/pits.21747

Bear, G. G., Mantz, L. S., Glutting, J. J., Chunyan, Y., Boyer, D. E., \& Suldo, S. (2015). Differences in bullying victimization between students with and without disabilities. School Psychology Review, 44(1), 98-116.

Bennett, A. (2014). Bullying: What you need to know. Kentucky English Bulletin, 64(1), 43-44.

Bhatnagar, N., \& Das, A. (2014). Attitudes of secondary regular schoolteachers toward inclusive education in New Delhi, India: A qualitative study. Exceptionality Education International, 24(2), 17-30.

Bosworth, K., \& Judkins, M. (2014). Tapping into the power of school climate to prevent bullying: One application of school-wide positive behaviour interventions and supports. Theory into Practice, 53(4), 300-307.

Bronfenbrenner, U. (1993). Ecological models of human development. In T. Husen \& T. N. Postlethwaite (Eds.), The International Encyclopedia of Education (2nd ed., pp. 1643-1647). New York, NY: Elsevier Science.

Bronfenbrenner, U. (1988). The ecology of cognitive development: Research models and fugitive findings. In R. H. Wozniak \& K. W. Fischer (Eds.), Development in Context: Acting and Thinking in Specific Environments (pp. 3-44). Hillsdale, JN: Erlbaum.

Bronfenbrenner, U. (1986). Ecology of the family as a context for human development: Research perspectives. Developmental Psychology, 22(6), 723-742.

Bronfenbrenner, U. (1979). The Ecology of Human Development: Experiments by Nature and Design. Cambridge, MA: Harvard University Press.

Bronfenbrenber, U. (1977). Toward an experimental ecology of human development. American Psychologist, 32(7), 513- 530.

Bronfenbrenner, U. (1974). Developmental research, public policy, and the ecology of childhood. Child Development, 45(1), 1-5. 
Bullying and Statistics (2012). Retrieved 12 January 2017, from http:/www.martialarts forpeace.com/pages/bullyingstatistics.html

Caldwell, B. (2012). Twenty-five to life for adolescent mistakes: Juvenile strikes as cruel and unusual punishment. University Of San Francisco Law Review, 46(3), 581-653.

Charmaraman, L., Jones, A. E., Stein, N., \& Espelage, D. L. (2013). Is it bullying or sexual harassment? Knowledge, attitudes, and professional development experiences of middle school staff. Journal of School Health, 83(6), 438-444. doi: 10.1111/ josh.12048

Chui, W., \& Chan, H. (2015). Self-control, school bullying perpetration, and victimization among Macanese adolescents. Journal of Child \& Family Studies, 24(6), 1751-1761. doi: 10.1007/s10826-014-9979-3

Ciudin, M., \& Turliuc, M. N. (2016). Predictors of psychosocial adjustment in adolescents with chronically ill parents: Testing the moderating role of family functioning and perceived social support. International Journal of Education \& Psychology in the Community, 6(1/2), 7-24.

De Souza, M., \& McLean, K. (2012). Bullying and violence: Changing an act of disconnectedness into an act of kindness. Pastoral Care in Education, 30(2), 165-180.

Deaton, S. (2015). Social learning theory in the age of social media: Implications for educational practitioners. Journal of Educational Technology, 12(1), 1-6.

Dehkordi, L. M., Babashahi, M., \& Irajpour, A. (2016). Nonprofessional care in chronic critically ill patient: A qualitative study. International Journal of Preventive Medicine, 1-5. doi: 10.4103/2008-7802.195209

Diers, J. (2013). Why the world needs to get serious about adolescents: A view from UNICEF. Journal of Research on Adolescence (Wiley-Blackwell), 23(2), 214-222. doi: 10.1111/jora.12042

Douglas, E. M. (2014). A comparison of child fatalities by physical abuse versus neglect: Child, family, service, and worker characteristics. Journal of Social Service Research, 40(3), 259-273. doi: 10.1080/01488376.2014.893948

Englander, M. (2012). The interview: Data collection in descriptive phenomenological research. Journal of Phenomenological Psychology 43, 13-35. Retrieved 10 December 2016, from http://phenomenologyblog.com/wp-content/uploads/2012/ 04/Englander-2012-The-Interview-Data-Collection-in-Descriptive-PhenomenologicalHuman-Scientific-Research.pdf

Espelage, D. L. (2014). Ecological theory: Preventing youth bullying, aggression, and victimization. Theory into Practice, 53(4), 257-264. doi:10.1080/00405841.2014. 947216

Foster, H., \& Brooks-Gunn, J. (2013). Neighbourhood, family and individual influences on school physical victimization. Journal of Youth \& Adolescence, 42(10), 15961610 .

Garthe, R. C., Sullivan, T. N., \& Kliewer, W. (2015). Longitudinal relations between adolescent and parental behaviours, parental knowledge, and internalizing behaviours among urban adolescents. Journal of Youth \& Adolescence, 44(4), 819-832. doi: 10.1007/s10964-014-0112-0

Geller, J., Avis, J., Srikameswaran, S., Zelichowska, J., Dartnell, K., Scheuerman, B., \& ... Ball, G. (2015). Developing and pilot testing the readiness and motivation interview for families in pediatric weight management. Canadian Journal of Dietetic Practice \& Research, 76(4), 190-193. doi: 10.3148/cjdpr-2015-024 
Golmaryami, F. N., Frick, P. J., Hemphill, S. A., Kahn, R. E., Crapanzano, A. M., \& Terranova, A. M. (2016). The social, behavioural, and emotional correlates of bullying and victimization in a school-based sample. Journal of Abnormal Child Psychology, 44(2), 381-391. doi: 10.1007/s10802-015-9994-x

Hertz, M. F., Everett Jones, S., Barrios, L., David-Ferdon, C., \& Holt, M. (2015). Association between bullying victimization and health risk behaviors among high school students in the United States. Journal of School Health, 85(12), 833-842. doi: 10.1111/josh.12339

Hoglund, W. G., Hosan, N. E., \& Leadbeater, B. J. (2012). Using your WITS: A 6-year follow-up of a peer victimization prevention program. School Psychology Review, 41(2), 193-214.

Hong, J., \& Garbarino, J. (2012). Risk and protective factors for homophobic bullying in schools: An application of the social-ecological framework. Educational Psychology Review, 24(2), 271-285. Retrieved 15 January 2017, from http://www.state. nj.us/education/students/safety/behavior/hib/

Huang, H., Hong, J., \& Espelage, D. (2013). Understanding factors associated with bullying and peer victimization in Chinese schools within ecological contexts. Journal of Child \& Family Studies, 22(7), 881-892.

Jansen, P., Verlinden, M., Dommisse-van Berkel, A., Mieloo, C., van der Ende, J., Veenstra, R., \& ... Tiemeier, H. (2012). Prevalence of bullying and victimization among children in early elementary school: Do family and school neighbourhood socioeconomic status matter? BMC Public Health, 12, 494. doi: 10.1186/14712458-12-494

Jiang, L., Chen, H., Ouyang, Y., \& Li, C. (2015). A multisource retrospective audit method for data quality optimization and evaluation. International Journal of Distributed Sensor Networks, 2015,1-8. doi: 10.1155/2015/195015

Johnston, P., Tankersley, M., Joenson, T., Hupp, M., Buckley, J., RedmondMcGowan, M., \& ... Walsh, A. (2014). Motivations behind "bullies then offenders" versus "pure bullies": further suggestions for anti-bully education and practice. Education, 134(3), 316-325.

Jones, J. R., \& Augustine, S. M. (2015). Creating an anti-bullying culture in secondary schools: Characteristics to consider when constructing appropriate anti-bullying programs. American Secondary Education, 43(3), 73-84.

Kearney, C., \& Graczyk, P. (2014). A response to intervention model to promote school attendance and decrease school absenteeism. Child \& Youth Care Forum, 43(1), 1-25. doi: 10.1007/s10566-013-9222-1.

Klomek, A. B., Kopelman-Rubin, D., Al-Yagon, M., Berkowitz, R., Apter, A., \& Mikulincer, M. (2016). Victimization by bullying and attachment to parents and teachers among student who report learning disorders and/or attention deficit hyperactivity disorder. Learning Disability Quarterly, 39(3), 182-190. doi: 10.1177/ 0731948715616377

Koskey, K. K. (2016). Using the cognitive pretesting method to gain insight into participants' experiences: An illustration and methodological reflection. International Journal of Qualitative Methods, 15(1), 1-13. doi: 10.1177/1609406915624577

Kramer, A. J. (2015). One strike and you're out: The application of labelling theory to the New Jersey anti-bullying bill of rights act. Seton Hall Law Review, 45(1), 261284. 
Kueny, M. T., \& Zirkel, P. A. (2012). An analysis of school anti-bullying laws in the United States. Middle School Journal (J3), 43(4), 22-31.

Lantos, J., \& Halpern, J. (2015). Bullying, social hierarchies, poverty, and health outcomes. Pediatrics, 135, S21-S23. doi: 10.1542/peds.2014-3549B

Leeuwis, F. H., Koot, H. M., Creemers, D. M., \& van Lier, P. C. (2015). Implicit and explicit self-esteem discrepancies, victimization and the development of late childhood internalizing problems. Journal of Abnormal Child Psychology, 43(5), 909919. doi: 10.1007/s10802-014-9959-5

Liang, Y., Ferdinando, H., Seppänen, T., \& Alasaarela, E. (2014). Physical violence detection for preventing school bullying. Advances in Artificial Intelligence (16877470), 1-9. doi: 10.1155/2014/740358

Malecki, C., Demaray, M., Coyle, S., Geosling, R., Rueger, S., \& Becker, L. (2015). Frequency, power differential, and intentionality and the relationship to anxiety, depression, and self-esteem for victims of bullying. Child \& Youth Care Forum, 44(1), 115-131. doi: 10.1007/s10566-014-9273-y

Margeviciute, A. (2016). Definition of bullying in compulsory education from general to legal perspective. Public Security \& Public Order / Visuomenes Saugumas Ir Viesoji Tvarka, 17, 83-103.

Mason, D. M., \& Ide, B. (2014). Adapting qualitative research strategies to technology savvy adolescents. Nurse Researcher, 21(5), 40-45. doi: 10.7748/nr.21.5.40.e1241

Morgan, L. 1., Leatzow, A., Clark, S., \& Siller, M. (2014). Interview skills for adults with autism spectrum disorder: A pilot randomized controlled trial. Journal of Autism \& Developmental Disorders, 44(9), 2290-2300. doi: 10.1007/s10803-014-2100-3

Nash, L. (2012). New Jersey's anti-bullying fix: A solution or the creation of an even greater first amendment problem? Brigham Young University Law Review, 2012(3), 1039-1070.

O’Neill, M., Calder, A., \& Allen, B. (2014). Tall poppies: Bullying behaviours faced by Australian high-performance school-age athletes. Journal of School Violence, 13(2), 210-227.

Ofe, E. E., Plumb, A. V., Plexico, L. W., Haaka, N. J., Nippold, M., \& Kelly, E. (2016). School-based speech-language pathologists' knowledge and perceptions of autism spectrum disorder and bullying. Language, Speech \& Hearing Services in Schools, 47(1), 59-76. doi: 10.1044/2015_LSHSS-15-0058

Paat, Y. (2013). Working with immigrant children and their families: An application of Bronfenbrenner's ecological systems theory. Journal of Human Behaviour in the Social Environment, 23(8), 954-966. doi: 10.1080/10911359.2013.800007

Palinkas, L., Horwitz, S., Green, C., Wisdom, J., Duan, N., \& Hoagwood, K. (2015). Purposeful sampling for qualitative data collection and analysis in mixed method implementation research. Administration \& Policy in Mental Health \& Mental Health Services Research, 42(5), 533-544. doi: 10.1007/s10488-013-0528-y

Patton, D., Hong, J., Williams, A., \& Allen-Meares, P. (2013). A review of research on school bullying among African American youth: An ecological systems analysis. Educational Psychology Review, 25(2), 245-260.

Patton, M. Q. (2002). Qualitative research and evaluation methods (3rd ed.). Thousand Oaks, CA: Sage.

Pister, R. (2014). Understanding bullying through the eyes of youth. Journal of Ethnographic \& Qualitative Research, 9(1), 27-43. 
Ramirez, O. O. (2013). Survivors of school bullying: A collective case study. Children \& Schools, 35(2), 93-99.

Rigby, K. (2014). How teachers address cases of bullying in schools: a comparison of five reactive approaches. Educational Psychology in Practice, 30(4), 409-419. doi: 10.1080/02667363.2014.949629

Ringwalt, C., \& Shamblen, S. (2012). Is there an association between adolescent bullying victimization and substance abuse? Journal of Drug Education, 42(4), 447-467. doi: 10.2190/DE.42.4.e

Rostkowski, S. M., \& Singh, R. K. (2015). Managing through social ostracization in the workplace. Ethics \& Critical Thinking Journal, 2015(3), 72-111.

Saldana, L. (2015). An integrated intervention to address the comorbid needs of families referred to child welfare for substance use disorders and child neglect: Fair pilot outcomes. Child Welfare, 94(5), 167-186.

Schneider, S., Odonnell, L., \& Stueve, A. S. (2012). Cyber-bullying, school bullying, and psychological distress: A regional census of high school students. American Journal of Public Health, 102(1), 171-177. doi: 10.2105/AJPH.2011.300308

Singh, A. A. (2013). The use of popular opinion leader (POL) groups and the reduction of "gay bullying" in middle school: A case study inquiry of group leader experiences. Journal for Specialists in Group Work, 38(3), 184-206. doi: 10.1080/01933922. 2013.800177

Snelgrove, S. R. (2014). Conducting qualitative longitudinal research using interpretative phenomenological analysis. Nurse Researcher, 22(1), 20-25.

State of New Jersey, Department of Education (2013). Harassment, intimidation, and bullying (HIB), 1996-2010. Retrieved 12 January 2017, from http:/www.state.nj. us/education/students/safety/behavior/hib/

Swearer, S. M., Wang, C., Berry, B., \& Myers, Z. R. (2014). Reducing bullying: Application of social cognitive theory. Theory into Practice, 53(4), 271-277. doi: 10.1080/ 00405841.2014 .947221

Teicher, M. H., \& Parigger, A. (2015). The 'maltreatment and abuse chronology of exposure' (MACE) scale for the retrospective assessment of abuse and neglect during development. Plos One, 10(2), 1-37. doi: 10.1371/journal.pone.0117423

Topcu, C. \& Erdur-Baker, O. (2012). Affective and cognitive empathy as mediators of gender differences in cyber and traditional bullying. School Psychology International, 33(5), 550-561.

Van Fleet, D. \& Van Fleet, E. (2012). Towards a behavioural description of managerial bullying. Employee Responsibilities \& Rights Journal, 24(3), 197-215. doi: 10.1007/ s10672-012-9190-x

Wilder, K. (2014). Stop bullying me!. Kentucky English Bulletin, 64(1), 48-49.

Williams, K. \& Kennedy, J. H. (2012). Bullying behaviours and attachment styles. North American Journal of Psychology, 14(2), 321-337.

Yoo, J. H. (2016). The effect of professional development on teacher efficacy and teachers' self-analysis of their efficacy change. Journal of Teacher Education for Sustainability, 18(1), 84-94. doi: 10.1515/jtes-2016-0007

Zamawe, F. C. (2015). The implication of using NVivo software in qualitative data analysis: Evidence-based reflections. Malawi Medical Journal: The Journal of Medical Association of Malawi, 27(1), 13-15. 
Zwierzynska, K., Wolke, D., \& Lereya, T. S. (2013). Peer victimization in childhood and internalizing problems in adolescence: A prospective longitudinal study. Journal of Abnormal Child Psychology, 41(2), 309-323.

Correspondence concerning this paper should be addressed to Lucy M. George Lekunze, 187 Oakwood Avenue, Orange NJ 07050, United States of America. Email: lucylekunze@gmail.com

\section{Appendix 1}

\section{Semi-Structured Questionnaire}

1. What types of bullying actions have you detected during this academic year?

2. What are examples of events you witnessed and intervened during this academic year?

3. What different forms of bullying have you witnessed throughout your career as a teacher?

4. What types of bullying/victimisation behaviours are common among girls? What types are common among boys?

5. How have the bullying behaviours exhibited by different genders evolved over time?

6. How often are the bullying and victimisation behaviours exhibited?

7. How serious do you perceive the problem? Can you explain with examples?

8. What types of aggressive behaviours do you or do you not perceive as bullying?

9. What are the different forms of interventions that you use in this school?

10. How adequate or inadequate were the different intervention strategies?

11. How has professional training in bullying intervention (if any) helped you in bullying mitigation?

12. How involved are teachers in this school in the improvement of bullying-prevention policies and practices?

13. How secure do students feel to report abuse without any pressure from teachers?

14. What are often your immediate reactions when you witness a bullying encounter?

15. What is your biggest challenge with respect to managing students' bullying and victimisation behaviour?

16. What are your suggestions to address better bullying issues among students?

17. What artefacts or unrestricted document do you have to share? 